\title{
The relationship between visual acuity and the spatial duty cycle of periodic stimuli*
}

\author{
C. R. CAVONIUS \\ The Psychological Laboratory, University of Cambridge, Cambridge CB2 3EB, England
}

\begin{abstract}
Human visual acuity was measured with rectangular-wave gratings that had duty cycles (the proportion of each grating period that is light) that varied from 0.020 to 0.975 . When adaptation level is held constant, the results are consistent with the hypothesis that only the amplitude of the fundamental Fourier component is detected at threshold.
\end{abstract}

In 1937, Shlaer measured visual acuity with rectangular-wave gratings in which the duty cycle (the fraction of each grating period that is occupied by a light bar) varied from 0.50 to 0.875 , and found that visual acuity remained practically constant as the duty cycle was changed. Because the contrast of gratings changes when the duty cycle is changed (this is discussed in more detail later, and in Fig. 1), the amplitude of the fundamental frequency (first Fourier component) of an image of these gratings also changes with duty cycle. Shlaer's results thus appear to differ from those in a more recent report by Campbell and Robson (1968), who found that the visibility of periodic gratings depends upon the amplitude of their fundamental component. For example, Campbell and Robson demonstrated that (except at low spatial frequencies) the sensitivity of the visual system to gratings that have sine-wave, rectangular-wave, and sawtooth luminance profiles is identical when the amplitudes of the fundamental components of the gratings are made equal by adjusting the modulation depth of the gratings. In the present experiment, visual acuity was measured over a wider range of duty cycles than that used by Shlaer, in order to attempt to resolve the difference between his conclusions, which were based on visual acuity measured with high-contrast gratings, and Campbell and Robson's, which were based on experiments in which the sensitivity of the visual system was measured with test objects that had relatively low contrasts.

The types of stimuli that were used are shown schematically in Fig. 1. The width of one period was the same for all of the gratings $(1.6 \mathrm{~mm})$, but the gratings differed in the relative widths of the dark and light bars. In order to be consistent with current usage, contrast is defined in Fig. 1 as the ratio of the difference between the luminance of the light and

*Supported by the James McKeen Cattell Fund. I am grateful to J. G. Robson for advice in interpreting the data, to F. W. Campbell for critically reading the manuscript, and to Professor $O$. L. Zangwill for providing facilities. dark bars, divided by twice the mean luminance. If the luminance of the dark bars is zero, $m$ becomes $1 / 2 \beta$, and $a_{0}$ is simply $2 \sin \beta \pi / \beta \pi$. Thus, the amplitude of the fundamental component increases monotonically as duty cycle is decreased.

\section{METHOD}

Stimuli were transparent prints of rectangular-wave gratings that were seen by the $O$ as a $22 \times 22 \mathrm{~mm}$ square field of horizontal bars, centered in a homogeneous $100 \times 100 \mathrm{~mm}$ square surround. Both stimulus and surround were illuminated from behind by light from an incandescent lamp, diffused by a sheet of opal acrylic. Large changes in stimulus luminance were made with neutral density filters and small changes by adjusting the lamp voltage. The surround luminance was $10 \%$ that of the space-average luminance of the grating. A surround that is this bright is adequate to eliminate the disturbing halo that appears around a grating that is viewed in the dark. Slightly higher acuity might have been measured if the surround had been brighter, but it is unlikely that this would have had any effect on the relative values of acuity that were measured with different duty cycles.

Those gratings that had duty cycles between 0.30 and 0.80 were contact prints on high-contrast film of Letratone Pattern LT 933. Stimuli that had higher and lower duty cycles were made by photographing large gratings made of drafting tapes on white paper. The 0.975-duty-cycle grating was made by stretching $0.04-\mathrm{mm}$ wires across a clear plastic base, and the 0.02 -duty-cycle grating was made by scribing clear lines through the emulsion of an exposed and developed photographic plate. The duty cycle and period of the gratings were measured with a microscope and calibrated reticle. The contrast between the light and dark bars was very high; the dark bars had an optical density greater than 4.0.

In order to maintain a constant adaptation level, the luminance of the gratings was adjusted to maintain a mean luminance equivalent to a uniform $100-\mathrm{cd} / \mathrm{m}^{2}$ field. (One exception to this is described below.) For example, if the duty cycle was halved, the luminance of the light bars was doubled so that the flux reaching the $O$ remained constant.

To measure visual acuity, the size of the test objects was held constant and the viewing distance was varied. Because of the wide range of viewing distances (almost $5 \mathrm{~m}$ ) that was needed, this was done by moving the $O$ rather than by moving the stimuli. The $O$ approached the stimuli until he could detect the presence of a grating, and was allowed to establish his own criterion for detection. Since the size of the test field was constant, the size of its retinal image changed with viewing distance. This should not have much effect on the results since the visibility of high-frequency gratings is scarcely influenced by field size (Cavonius \& Hilz, 1973). 
Luminance Profile

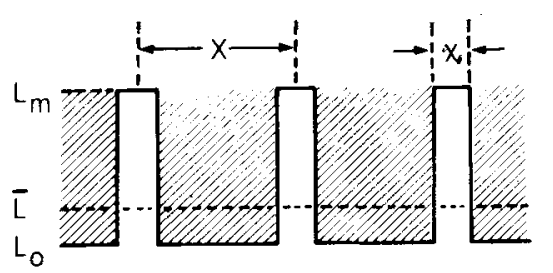

¿

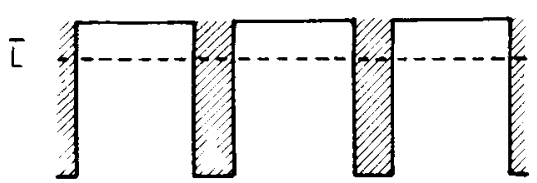

Duty Cycle

$\beta=x / x$

.25
Contrast

$$
m=\frac{L_{m}-L_{O}}{2 \bar{L}}
$$

2
Fundamental Amplitude

$$
a_{0}=\frac{4 m \sin \beta \pi}{\pi}
$$

1.8

Fig. 1. Luminance distributions of three rectangular gratings, and definitions of duty cycle, contrast, and fundamental amplitude. In the calculations, $L_{0}=0$. For simplicity, the parameter that describes the duty cycle $(\chi)$ has been defined as the width of one light bar instead of half the width, as is conventional in time-series analysis.

Three experienced Os were used. P.W. and D.C. were emmetropic, and J.M., who is 4D myopic, wore his usual correction. Observations were made binocularly with natural pupils, which were between 5 and $6 \mathrm{~mm}$ in diam.

\section{RESULTS}

When visual acuity is measured with grating targets, it is conventionally expressed as the reciprocal of the visual angle (in minutes of arc) that is subtended at threshold by a half period of the grating. For gratings that have a duty cycle of 0.50 , this is equivalent to the reciprocal of the angle subtended by one dark bar. Except in Fig. 3, this convention was used in the present study, although it is an arbitrary definition in the case of gratings in which the dark and light bars are not equally wide.

The filled symbols in Fig. 2 show acuity values that were measured with different duty cycles at a constant mean adaptation level of $100 \mathrm{~cd} / \mathrm{m}^{2}$. The three Os differed in the distance at which they detected all gratings, possibly as a result of the criteria they used. The lower acuity of J.M. is at least in part caused by the $-4 \mathrm{D}$ correction that he wore, which reduced the size of the retinal image of the test gratings. The differences in sensitivity among Os remained constant as the duty cycle changed, so that the shapes of the acuity vs duty-cycle functions are similar and can be fitted by the same model, which will be described

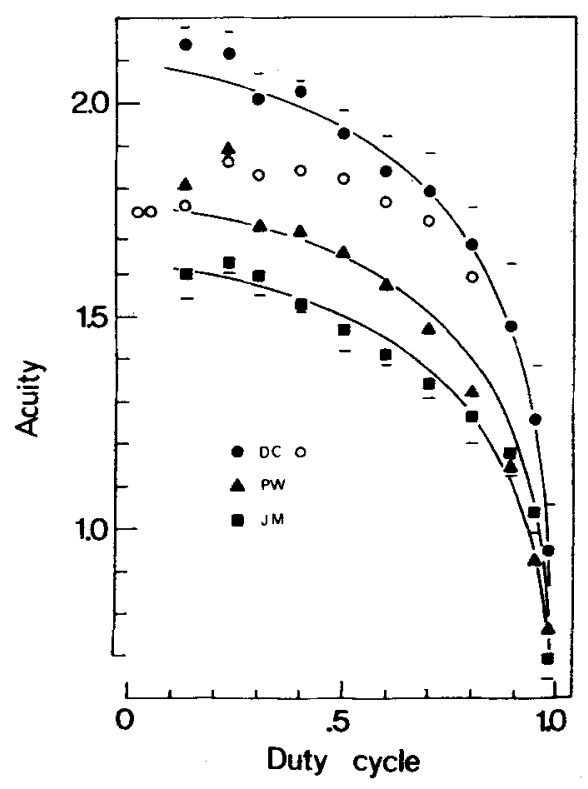

Fig. 2. Visual acuity as a function of duty cycle with three $O s$ at a constant adaptation level [filled symbols] and one $O$ with constant luminance [open circles]. Solid lines are the acuities calculated on the assumption that only the fundamental component is detected at threshold. The resulting values have been multiplied by 0.96 for J.M., by 1.06 for P.W., and by 1.25 for D.C. Horizontal lines above the data for D.C. and below those for J.M. show one standard deviation. P.W.'s standard deviations [which have been omitted for clarity] were about twice J.M.'s. 
later. Acuity increases monotonically as duty cycle is reduced. This effect is not restricted to one adaptation level, since the same shape function was obtained at adaptation levels of 100,10 , and $1 \mathrm{~cd} / \mathrm{m}^{2}$ (not shown). The filled symbols in Fig. 2 were obtained at a constant mean adaptation level. If, instead, the luminance of the light bars is held constant as duty cycle is varied, the results shown by the open circles are obtained. Acuity now declines somewhat at low duty cycles, although it is still quite high at a duty cycle of 0.02 , which was the lowest value that was used. Eventually, of course, acuity must drop precipitously when very low duty cycles are presented, for if luminance is held constant, the total flux that is emitted by the light bars will approach the threshold for light detection.

Acuity can also be defined as the reciprocal of the visual angle that is subtended by some critical detail of the stimulus pattern. This is usually the width of a dark area, such as the width of one stroke of a test letter. In Fig. 3, the constant adaptation-level data of Fig. 2 are replotted to show the width of a single dark bar of the grating at the detection threshold. The fact that the finest dark line that is visible is approximately proportional to the complement of the duty cycle is simply another way of saying that the visibility of gratings does not change drastically with changes in duty cycle.

\section{DISCUSSION}

Detection of small points of light on a dark background depends primarily on their luminance (Riggs, 1965). Figure 2 shows that this also tends to hold for the detection of thin light bars. When the width of a narrow bar is reduced, the illuminance of its retinal imagu decreases. However, if the luminance of the light bar is increased, the peak illuminance of the retinal image is restored and visibility is maintained.

A quantiative description of the visibility of gratings of different duty cycles can be made by applying Campbell and Robson's finding that the detectability of high-frequency gratings depends only on the amplitude of the fundamental frequency (the first Fourier component). As the duty cycle of a rectangular-wave grating is reduced, the amplitude of its fundamental inereases monotonically, as shown in Fig. 1. If the detectability of a grating depends on the amplitude of the fundamental, it should be independent of the way in which this amplitude is generated, so that the results for different wave forms and contrasts should be similar. Campbell and Robson (1968) measured the contrast that is needed to detect $500 \mathrm{~cd} / \mathrm{m}^{2}$ sine-wave gratings of various spatial frequencies. In order to apply their data to the present study, the fundamental amplitude of rectangularwave gratings with duty cycles 0.05 to 0.98 was calculated, and the spatial frequencies at which sine-wave gratings with these amplitudes could be

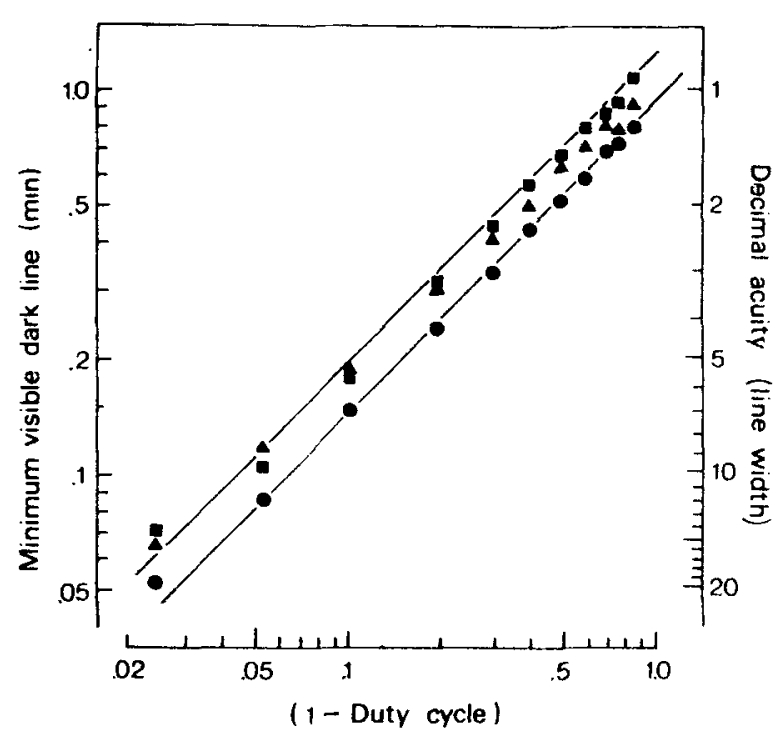

Fig. 3. Visual acuity, defined as the reciprocal of the visual angle subtended by each dark bar of the grating, as a function of duty cycle. The upper line shows the predicted values for J.M., assuming that only the fundamental is detected at threshold, and the lower line, the predicted values for D.C. The predicted function for P.W., which is close to that for J.M, has been omitted for clarity.

resolved were taken from Campbell and Robson's Fig. 2. [Their measurements extend to contrasts of only about 0.6 , but their high-frequency data can be matched very closely by the empirical model $\mathrm{m}=$ $\exp (0.15 v-7)$, where $v$ is the spatial frequency of the grating. This formula was used to extrapolate their data to higher contrasts.] The curves in Fig. 2 are the values predicted from the Campbell and Robson data, multiplied by the individual scaling factors that are given in the figure caption. The agreement between prediction and data is rather good for all Os. If there is a systematic difference, it is that the predicted values are slightly lower than the measured data at low duty cycles and slightly higher at duty cycles near 0.7 . The agreement between the results of two rather different experiments tends to support the hypothesis that the detection of gratings depends on the fundamental frequency of the grating. Even in the case of very low duty cycles, for which the amplitudes of all harmonics become nearly the same, only the fundamental will contribute to the detection of the grating at threshold, because the sensitivity of the visual system drops rapidly at high spatial frequencies, and the spatial frequencies of the higher harmonics in these gratings are too high to be detected. This is supported by the subjective observation that all the gratings looked very similar at threshold.

In these data, the influence of duty cycle on acuity is greater than that reported by Shlaer (1937), who found that an increase in duty cycle from 0.50 to 0.875 caused acuity to drop by $5 \%$, whereas in the present study the same increase caused a $20 \%$ change in 
acuity. One methodological difference between the two studies is that Shlaer used a constant background luminance, so that the adaptation level was proportional to the duty cycle. However, as shown by the open circles in Fig. 2, this has little effect on the detection of gratings that have duty cycles that are greater than 0.5 , and is not sufficient to explain the difference between the results of the two studies.

Another difference between the studies is that Shlaer used a $2-\mathrm{mm}$ artificial pupil, which sets a Rayleigh limit to resolution that corresponds to a visual acuity of 1.7 (Riggs, 1965). This is precisely the maximum grating acuity that Shlaer found. In the present experiment, natural pupils were used, so that diffraction did not set an upper limit to acuity, which may have allowed the effect of changing contrast to be seen.

It should be emphasized that these results do not necessarily support the popular notion that the visual system is organized into channels that are sensitive to narrow bands of spatial frequencies, for the same results would result if the gratings had been imaged by a passive system that had a modulation transfer function that was similar to the high-frequency branch of the contrast-sensitivity vs spatial-frequency characteristic of the human visual system. Differences between the predictions of spatial-frequency-channel models and receptive-field models tend to occur in the low spatial-frequency region, and would not be expected to show up under the high-frequency, high-contrast conditions that were used in this experiment.

\section{REFERENCES}

Camprell, F. W., \& Rosson, J. G. Application of Fourier analysis to the visibility of gratings. Journal of Physiology. $1968,197,551-566$.

Cavonius, C. R., \& Hilz, R. Invariance of visual receptive fields and visual acuity with viewing distance. Journal of the Optical Society of America, 1973, 63, 929-933.

Riggs, L. A. Visual acuity. In C. H. Graham (Ed.). Vision and visual perception. New York: Wiley, 1965.

SHLAER, S. The relation between visual acuity and illumination. Journal of General Physiology, 1937, 21, 165-188.

(Received for publication September 1973; revision accepted February 28, 1974.) 